Psychotherapeut 2021 · 66:518-523 https://doi.org/10.1007/s00278-021-00536-z Angenommen: 3. August 2021 Online publiziert: 20. September 2021

(c) Der/die Autor(en) 2021

\section{Psychische Gesundheit von Contergan-geschädigten Menschen}

\section{Der traumatische Schatten pränataler Thalidomidexposition}

\author{
Alexander Niecke \\ Klinik und Poliklinik für Psychosomatik und Psychotherapie, Medizinische Fakultät und Uniklinik, \\ Universität zu Köln, Köln, Deutschland
}

Hintergrund: Sechs Jahrzehnte nach der pränatalen pharmakogenen Schädigung durch Thalidomid prägen vielfältige biopsychosoziale Folgeschädigungen das klinische Bild von Menschen mit Contergan-Behinderungen.

Fragestellung: Wie lässt sich das erst in der zweiten Lebenshälfte sichtbar gewordene hohe Ausmaß psychischer Störungen näher verstehen?

Material und Methode: Literaturüberblick zum aktuellen Stand der empirischen Forschung mit Diskussion weiterführender Fragen.

Ergebnisse: Menschen mit thalidomidinduzierten Schwerstbehinderungen zeigten jahrzehntelang bemerkenswerte Kompensationsleistungen. Ein sehr hoher Bildungsstatus sowie eine gute berufliche und soziale Integration wurden über viele Jahre erreicht. In der jüngeren Vergangenheit ist es jedoch zur zunehmenden sozialen Desintegration gekommen. Erstmals wurde eine hohe Rate psychischer Störungen, bei gleichzeitig bestehenden Barrieren im Zugang zur psychotherapeutischen Versorgung, beschrieben.

Schlussfolgerung: Das große Vorkommen psychischer Störungen im Alter kann einerseits als durch multidimensionale, teilweise bereits pränatal erworbene Traumatisierungen verursacht, andererseits als eine lange Zeit zugunsten einer Monostigmatisierung bestehende selektive Nichtwahrnehmung des Psychischen verstanden werden. Nicht nur für die Betroffenen ist es wichtig, dass der Fall Contergan im kollektiven Gedächtnis erinnert wird.

\title{
Schlüsselwörter
}

Thalidomid · Embryopathie · Körperbehinderung · Traumatisierung · Therapeutisches Erinnern

Das Wort Contergan ist der Inbegriff für die bis heute folgenschwerste Arzneimittelkatastrophe der deutschen Geschichte (Kirk 1999). Dieser lässt unweigerlich an schwerstbehinderte Menschen aufgrund einer pränatal erworbenen pharmakogenen Schädigung denken (Lehnhard-Schramm 2016) und gilt zudem als tragisches Beispiel für das Versagen von Staat, Ärzteschaft und Wissenschaft (Thomann 2005). Die vorliegende Arbeit zeichnet den historischen Kontext des Falls Contergan nach, verschafft einen Überblick über den aktuellen Stand der
Forschung der biopsychosozialen Langzeitfolgen und diskutiert offene Fragen.

\section{Historischer Kontext}

In Westdeutschland waren die 1950erJahre vom enormen Wirtschaftsboom der Nachkriegsjahre geprägt („Wirtschaftswunder"). Eine mächtige pharmazeutische Industrie entwickelte neue hochwirksame Medikamente, wobei die Vermarktung von Arzneimitteln noch einen praktisch gesetzesfreien, vorpolitischen Raum darstellte (Müller-Oerlinghausen 2005). In den Labors der Fa. Chemie Grünenthal 
wurde 1954 die Substanz Thalidomid (N-Phthalyglutaminsäureimid) synthetisiert. Thalidomid wirkte beruhigend und hatte in seiner vermeintlichen Atoxizität den entscheidenden Vorteil gegenüber den damals verfügbaren Barbituraten, die aufgrund ihrer geringen therapeutischen Breite zu den letalen Medikamenten gezählt werden (Peters 2012).

Contergan wurde 1957 als rezeptfreies Sedativum zugelassen. Die vermeintliche Harmlosigkeit und Unschädlichkeit führte zu der gezielten Marketingstrategie "sicherer als andere" zu sein (Spiegel vom 16.08.1961: „Zuckerplätzchen forte“). So eroberte Contergan in kurzer Zeit den Arzneimittelmarkt, wurde nach Aspirin zum zweitmeistverkauften Pharmakon (Lehnhard-Schramm 2016) und avancierte zum Lifestyle-Medikament einer ganzen Generation (Niecke et al. 2017). Die sedierenddistanzierenden Eigenschaften von Contergan trafen gewissermaßen auf eine traumatisierte und entwurzelte Gesellschaft der Nachkriegsjahre (Osten 2019).

Das Indikationsspektrum war breit, es beinhaltete u. a. schwangerschaftsbedingte Übelkeit. Selbst als bereits ab 1959 erste Hinweise auf neurotoxische Wirkungen bei der Herstellerfirma eingingen, gelang es, die Einführung der Rezeptpflicht bis in den Herbst 1961 zu verhindern (Thomann 2005). Vor dem Hintergrund wirtschaftlicher Interessen und unzureichend ausgebildeter staatlicher Kontrollsysteme führte ein sorgloser Umgang mit einem fortschrittlichen Medikament, das nicht so harmlos war wie angenommen, schließlich zur Katastrophe. Allein in Westdeutschland wurden bis 1962 geschätzt 5000 sog. Contergan-Kinder geboren. Die Säuglingssterblichkeit bei Thalidomidembryopathie beträgt rund $40 \%$, was auf schwere Fehlbildungen der inneren Organanlagen zurückgeführt wurde (Smithells und Newman 1992). Die Zahl der damaligen Spontanaborte und Totgeburten ist eine unbekannte Dunkelziffer geblieben (Lenz 1988).

Vielfältige Gründe führten dazu, dass es erst 4 Jahre später gelang, die verheerende Embryotoxizität thalidomidhaltiger Präparate zu identifizieren und das Pharmakon 1961 vom deutschen Markt zurückzuziehen. Kurze Zeit nach der Marktrücknahme erfolgten erste Veröffentlichungen von McBride (1961) und Lenz (1961), die die
Einnahme von Thalidomid in der Schwangerschaft als die verantwortliche Noxe für die kindlichen Fehlbildungen nahelegten. Die Offenlegung der Embryotoxizität und das Bekanntwerden des großen Schadens lösten nach der Marktrücknahme von Contergan einen Skandal aus, der alle anderen politischen Ereignisse in den Schatten stellen sollte, so auch den Bau der Berliner Mauer. In der Folge begannen ein weitreichender gesellschaftspolitischer Diskurs über eine Sensibilisierung der Bevölkerung für Umweltrisiken sowie die Thematisierungen der Chancengleichheit für Behinderte und eines verstärkten staatlichen Eingriffs in die Arzneimittelregulation (Lehnhard-Schramm 2016); und Deutschland sollte sein erstes Arzneimittelgesetz und ein eigenständiges Gesundheitsministerium erhalten (MüllerOerlinghausen 2005). Das 1968 gegen die Herstellerfirma eingeleitete Strafverfahren wurde nach 4 Prozessjahren, „wegen geringfügiger Schuld der Angeklagten und mangelnden öffentlichen Interesses" eingestellt, nachdem per außergerichtlichem Vergleichsvertrag 100 Mio. D-Mark in eine Stiftung eingezahlt wurden (Friedrich 2005). Der straffreie Ausgang des Alsdorfer Gerichtsprozesses gilt als weiterer Skandal (Gemballa 1993).

Thalidomid wurde weltweit in 46 Ländern vertrieben, außer in Westdeutschland v.a. in Großbritannien, Japan, Schweden und Kanada; allerdings nicht in den Vereinigten Staaten, weil es den dort vorhandenen Sicherheitsstandards nicht entsprach (Knightley et al. 1979). Obwohl Thalidomid 1962 aus dem größten Teil der Welt verbannt wurde, ist es nie gänzlich vom Weltmarkt verschwunden und erlebt in den letzten Jahren eine Renaissance (Vargesson 2009). Aufgrund seiner immunmodulatorischen, entzündungshemmenden und antiangiogenetischen Eigenschaften wird das Medikament unter strengen Vorschriften beispielsweise in der Behandlung von Lepra, multiplem Myelom, M.Crohn und "Human-immunodeficiencyvirus"(HIV)-Infektion verschrieben sowie in einer Reihe von klinischen Bedingungen untersucht (Matthews und McCoy 2003). Tragischerweise sind in einigen entwicklungsschwächeren Ländern, insbesondere Brasilien, in dem Thalidomid zur Behandlung von Lepra verwendet wird, erneute
Fälle von Thalidomidembryopathie aufgetreten (Kowalski et al. 2015). Daher ist die Thalidomidembryopathie nicht nur ein medizinisches Versorgungsproblem für alternde Menschen auf der nördlichen Hemisphäre, sondern auch eine gegenwärtige Gesundheitsbedrohung für junge Familien auf der südlichen Hemisphäre (Weinrich et al. 2018).

\section{Stand der Forschung: biopsycho- soziale Langzeitfolgen}

Etwa 3000 bis 4000 Thalidomidüberlebende leben derzeit auf der Welt (Hinoshita et al. 2019), davon 2259 in Deutschland (Conterganstiftung, persönliche Mitteilung [E-Mail] über die Zahl ihrer Leistungsempfänger, Stand am 20.10.2020). Mehrere Studien haben die biopsychosozialen Langzeitfolgen von Contergan-geschädigten Menschen systematisch untersucht und dabei deutlich gemacht, dass neben weitreichenden körperlichen Folgeschäden auch erhebliche psychosoziale Beeinträchtigungen nachweisbar sind (Kruse et al. 2012; Peters et al. 2015; Newbronner und Atkin 2018).

\section{Körperliche Ursprungs- und Folgeschädigungen}

Selbst die einmalige Einnahme einer thalidomidhaltigen Substanz während des ersten Schwangerschaftsdrittels führt im Uterus zur Thalidomidembryopathie. Als thalidomidinduzierte Ursprungsschäden gelten die typischen Hemmungsfehlbildungen der oberen und unteren Extremitäten (sog. Dysmelien), die unterschiedliche Schweregrade aufweisen können und oft bilateral-symmetrisch auftreten. Neben Fehlbildungen des Skeletts kommen auch Fehlbildungen der Sinnesorgane (insbesondere der Ohren) und der inneren Organanlagen sowie neurologische Störungen vor (Peters 2012). Auf somatischer Ebene prägen inzwischen chronische muskuloskeletale Schmerzen (Niecke et al. 2021) und Einschränkungen der Mobilität das Beschwerdebild; diese sind in der Folge jahrzehntelang betriebener unphysiologischer Überkompensationsbewegungen aufgetreten. Zusätzlich kommen zunehmend altersassoziierte internistische Krankheiten dazu, und es wird 
ein andauernd hoher Bedarf an individueller medizinischer und pflegerischer Versorgung beschrieben (Kruse et al. 2012; Peters et al. 2015).

\section{Beziehungs- und Arbeitsfähigkeit}

Contergan-geschädigte Frauen und Männer sind deutlich seltener als die gleichaltrige Allgemeinbevölkerung Lebenspartnerschaften eingegangen (Kruse et al. 2012). Seltenere Paarbindungen, erschwerte körperliche Voraussetzungen (z.B. Schädigungen im Reproduktionssystem) und die gemachte Erfahrung der existenziellen Verletzbarkeit einer Schwangerschaft sind Faktoren, die auf die Generativität eingewirkt haben. Zusätzlich wird der notwendige Assistenz- und Pflegebedarf, der bislang nahezu vollständig vom Laienhelfersystem übernommen wurde, heute durch das altersbedingte Ausscheiden der Elterngeneration und den relativen Mangel an Partnerschaften und Nachkommen oftmals nicht mehr gedeckt (Peters et al. 2015). Viele Betroffene haben über Jahrzehnte einen bemerkenswerten Grad an Selbstständigkeit, Kreativität und Produktivität erreichen können. So wurden sowohl ein sehr hoher Bildungsgrad als auch ein hoher Anteil an Berufstätigkeit und langjährige Berufsausübung erreicht. Aber doch: In den vergangenen beiden Jahrzehnten ist der Anteil von Personen, die vorzeitig aus dem Erwerbsleben ausgeschieden sind, massiv angestiegen (Kruse et al. 2012).

\section{Psychisches Befinden}

In Deutschland gibt mittlerweile fast jeder zweite Betroffene an, unter krankheitswertigen psychischen Störungen zu leiden das ist doppelt so häufig wie in der altersadjustierten Allgemeinbevölkerung (Niecke et al. 2017). Ähnliche Ergebnisse wurden in Japan erstbeschrieben (Imai et al. 2014). Auf syndromaler Ebene dominieren depressive, somatoforme und alkoholbezogene Störungen (Niecke et al. 2017); in Schweden wurde eine erhöhte Rate autistischer Störungen nachgewiesen (Stromland et al. 1994). Außerdem stellte sich heraus, dass deutliche Behandlungsbarrieren im psychotherapeutischen Versorgungssystem bestehen (Niecke et al. 2017).

\section{Diskussion}

Auf dem Boden der geschilderten wissenschaftlichen Evidenz zu den psychosozialen Langzeitfolgen bleiben folgende Fragen bislang unbeantwortet:

1. Warum wird das hohe Ausmaß psychischer Störungen bei Contergangeschädigten Frauen und Männern erst nach einer jahrzehntelangen Latenz öffentlich wahrgenommen?

2. Können psychische Störungen bei Contergan-geschädigten Menschen als posttraumatisches Phänomen verstanden werden?

3. Warum ist die fortwährende Erinnerung an den Fall Contergan von Bedeutung?

\section{Spätmanifestation psychischer Störungen?}

Auf dem Boden der beschriebenen körperlichen und soziobiografischen Befunde erscheint es nicht verwunderlich, dass viele Betroffene unter Störungen des psychischen Befindens leiden. Es verwundert vielmehr, dass dies erst spät bemerkt wurde. Eine Spätmanifestation psychischer Störungen lässt sich im Sinne des Vulnerabilität-Stressbewältigung-Modells (Zubin und Spring 1977) als kollektives Schwellenphänomen beschreiben: Die lebenslang bestehende Vulnerabilität einer körperlichen Schwerstbehinderung konnte über viele Jahre durch adaptive Bewältigungsmechanismen kompensiert werden. Durch neu hinzutretende körperliche Folgeschäden sowie psychosoziale Stressoren (z.B. Verlust von Autonomie und sozialer Teilhabe) wurde jedoch mittlerweile ein kritischer Schwellenwert überschritten, was sich kollektiv in sich spätmanifestierenden psychischen Störungen ausdrückt.

Wahrscheinlich ist, dass psychische Störungen schon in früheren Lebensjahren bestanden haben, vielleicht sogar lebenslang, jedoch im gesellschaftlichen Diskurs lange Zeit nicht wahrgenommen wurden. Aus ärztlichen Gutachten der 1970er-Jahre geht hervor, dass psychische Auffälligkeiten bereits im Kindesund Jugendalter festgestellt wurden, man jedoch durch eine Fokussierung auf psychische Belastungen den Erfolg einer progressiv ausgerichteten orthopädischen Rehabilitation gefährdet sah. Auch seitens der akademischen Medizin wurden kaum Anstrengungen unternommen, die psychische Gesundheit zu untersuchen, „weil nicht zu erwarten sei, dass sich Contergan-geschädigte Frauen und Männer hinsichtlich des Auftretens psychischer Störungen von der Allgemeinbevölkerung unterscheiden" (Kruse et al. 2012). Ebenso wie seitens der Ärzteschaft vorhandenes seelisches Leid offenbar ausgeblendet oder ignoriert wurde, ist es aber auch denkbar, dass die Betroffenen selbst lange Zeit psychische Belastungen nicht offen kommuniziert haben. So könnte es sein, dass die Anforderung, mit dem Stigma einer äußerlich sichtbaren Körperbehinderung zu leben, groß genug war und es vermieden wurde, zusätzlich mit dem Stigma einer psychischen Erkrankung in Berührung zu kommen. Ein solches Verdeckthalten des Seelischen kann als Ausdruck einer selektiven Wahrnehmung des Körperlichen verstanden werden. Vieles spricht dafür, dass lange Zeit das Licht der Aufmerksamkeit selektiv auf die Körperbehinderung gelenkt und seelisches Leid nicht kommuniziert wurde.

\section{Multidimensionale Traumatisierung}

Der im Kontext der Contergan-Epidemie immer wieder verwendete Begriff der Katastrophe (Friedrich 2005; Kirk 1999; Peters 2012) legt nahe, dass es sich um ein traumatisierendes Ereignis gehandelt hat. Dabei bilden die thalidomidinduzierten pränatalen Ursprungsschädigungen gewissermaßen einen primären, biologisch fixierten, traumatischen Kern. Um diesen Kern gruppieren sich vielfältige individuelle Belastungen, beispielsweise frühbiografisch stattgehabte operative Mehrfachinterventionen mit lang dauernden Hospitalisierungen und Trennung von den Primärpersonen. Eine leistungsberechtigende Anerkennung als Contergan-Schadensfall sollen heute alle durch pränatale Thalidomidexposition induzierten Schädigungen finden, die zum Zeitpunkt der Geburt vorlagen oder angelegt waren (Conterganstiftungsgesetz vom 09.03.2017). Psychische Störungen werden in der aktuellen Auslegung des Conterganstiftungsgesetzes nicht berück- 
sichtigt, sondern als sekundäre Reaktion auf ein Leben mit Schwerstbehinderung aufgefasst. Diese gesellschaftspolitische Haltung bildet den Ausgangspunkt zu der Frage, ob psychische Störungen nicht ebenfalls auf eine ursächliche vorgeburtliche Schädigung zurückgeführt werden können und bereits bei der Geburt vorlagen oder zumindest angelegt waren.

\section{Pränatale psychische Traumatisierung?}

In der westlichen Philosophietradition vertrat bereits Aristoteles in seiner „Zeugungstheorie" den Standpunkt, einer mit der Zeugung einsetzenden sukzessiven Beseelung der Leibesfrucht. Forschungen der modernen Embryologie konnten diesen Standpunkt untermauern. So wird heute der Beginn des individuellen menschlichen Lebens nahezu übereinstimmend mit der Befruchtung der Eizelle und der damit verbundenen Bildung eines neuen menschlichen Lebewesens mit eigener genetischer Identität, die sich von jener der Mutter und des Vaters unterscheidet, gesehen. Die für die menschliche Persönlichkeit spezifischen Bewusstseinsphänomene sind pränatal, aber auch längere Zeit postnatal noch nicht vorhanden, sie sind aber bereits angelegt (Maio 2017). Forschungen zur Epigenetik konnten beispielsweise zeigen, dass mütterlicher Stress beim Fetus das Gen des Glukokortikoidrezeptors durch Methylierung über Jahre hinweg so verändert, dass Kinder später verstärkt auf Stress reagieren (Oberlander et al. 2008).

Die Frage, ob die unvermeidbare Aufnahme des stillen Gifts Thalidomid über das plazentare Blut, das die Aussprossung der Extremitäten verhindert, für den Embryo als ein frühpsychisch-affektives Phänomen, im Sinne eines leiblichen Gefühls von Unbehagen (Schmitz 2011), verstanden werden kann, ist sicherlich spekulativ. In einer rückblickenden Bewertung macht ein Embryo in einer Situation der umfassenden Ausweglosigkeit hier bereits erstmals die elementare Erfahrung des Nichtmöglichen, die sein späteres Dasein begleiten wird. Und ein Fetus, dessen Hörsinn so getrübt ist, dass er keinen akustischen Kontakt zur Mutter aufnehmen kann, macht bereits intrauterin spezielle Interaktionserfahrungen, die sich auf sein späteres Be- ziehungserleben auswirken. Hier stoßen wir in einen Grenzbereich des naturwissenschaftlich Nachweisbaren. Ein ätiologischer Zusammenhang zwischen der intrauterinen Aufnahme der embryotoxischen Substanz Thalidomid und der Entwicklung von psychischen Störungen ist durch nichts bewiesen, dennoch ist ein Zusammenhang aber denkbar. Sowohl eine direkte neurovaskuläre oder neurotoxische Schädigung als auch negative affektive Leibeserfahrungen oder intrauterine Interaktionsstörungen können einen psychotraumatischen Kern einer vorpsychischen Matrix ausgebildet haben, was zu einer bei der Geburt angelegten Disposition für psychische Störungen beigetragen hat.

\section{Zur Bedeutung der Erinnerung}

Eine Betroffene drückte ihr Erleben, in Vergessenheit geraten zu sein, mit folgenden Worten aus:

Die Ärzte wissen nicht einmal mehr, was Contergan ist. Ein junger Arzt fragte mich, woher ich die Behinderung hätte, worauf ich ihm antwortete, dass das von Contergan komme, worauf er fragte, warum ich dieses Medikament überhaupt eingenommen hätte....

In der Kulturwissenschaft hat sich nach Assmann (2017) mit der Einführung des Begriffs der Erinnerungskultur im Umgang mit der Vergangenheitsbewältigung in der jüngeren Geschichte ein Paradigmenwechsel vollzogen. Anders als zuvor führt der Umgang mit der Geschichte heute nicht mehr durch das Vergessen, sondern "durch das Nadelöhr der Anerkennung, Erinnerung und Aufarbeitung". Mit dem Ziel einer gesellschaftlichen Integration als Grundlage für eine gemeinsame Zukunft wird das Erinnern gebraucht, um die Last der Vergangenheit abzutragen. In der psychosomatischen Medizin wird Erinnern als Therapie eingesetzt. So kann innerhalb eines psychotherapeutischen Prozesses durch therapeutisches Erinnern die Last der eigenen Biografie bewusstwerden. Erst wenn diese eigene Geschichtslast erinnert und anerkannt wird, kann sie im weiteren Prozess durchgearbeitet und dabei emotional entschärft werden - um sie schließlich umso besser hinter sich zu lassen. In der Psychotherapie geht es somit um ein Vergessen, ein Vergeben, ein Aussöhnen (Assmann 2017). Die Erinnerung an den Fall Contergan ist nicht nur für unser heutiges medizinisches Menschenbild von Bedeutung, sondern nicht zuletzt auch für die Überlebenden der Contergan-Katastrophe. Für sie sind beide Aspekte des Erinnerns existenziell - nicht in Vergessenheit zu geraten und sich auszusöhnen.

\section{Fazit für die Praxis}

- Mittlerweile dominieren körperliche Folgeschäden, insbesondere schmerzhafte Bewegungseinschränkungen, das klinische Beschwerdebild der Betroffenen. Zusätzlich drohen soziale Desintegration und die Dekompensation jahrelang bestehender Kompensationsmechanismen. Fast die Hälfte der Überlebenden der Contergan-Katastrophe gibt an, mittlerweile unter krankheitswertigen psychischen Störungen zu leiden; dem stehen Barrieren im psychotherapeutischen Versorgungssystem gegenüber.

- Es reicht nicht aus, das erst spät in der Öffentlichkeit wahrnehmbar gewordene hohe Ausmaß psychischer Störungen, ausschließlich als kollektive Spätmanifestation aufzufassen, sondern kann auch als eine jahrelang bestehende selektive Nichtwahrnehmung des Seelischen ("When you carry the light into one corner, you darken the rest"; Francis Bacon) verstanden werden.

- Die Erinnerung an den Fall Contergan ist für die Überlebenden der Contergan-Katastrophe und unser heutiges medizinisches Menschenbild von Bedeutung.

\section{Korrespondenzadresse}

Dr. med. Alexander Niecke

Klinik und Poliklinik für Psychosomatik und

Psychotherapie, Medizinische Fakultät und

Uniklinik, Universität zu Köln

50937 Köln, Deutschland

alexander.niecke@uk-koeln.de

Danksagung. Mein Dank gilt Tobias Nolte und Rainer Obliers für wertvolle Hinweise.

Funding. Open Access funding enabled and organized by Projekt DEAL. 


\section{Einhaltung ethischer Richtlinien}

Interessenkonflikt. A. Niecke gibt an, dass kein Interessenkonflikt besteht.

Für diesen Beitrag wurden vom Autor keine Studien an Menschen oder Tieren durchgeführt. Für die aufgeführten Studien gelten die jeweils dort angegebenen ethischen Richtlinien.

Open Access. Dieser Artikel wird unter der Creative Commons Namensnennung 4.0 International Lizenz veröffentlicht, welche die Nutzung, Vervielfältigung, Bearbeitung, Verbreitung und Wiedergabe in jeglichem Medium und Format erlaubt, sofern Sie den/die ursprünglichen Autor(en) und die Quelle ordnungsgemäß nennen, einen Link zur Creative Commons Lizenz beifügen und angeben, ob Änderungen vorgenommen wurden.

Die in diesem Artikel enthaltenen Bilder und sonstiges Drittmaterial unterliegen ebenfalls der genannten Creative Commons Lizenz, sofern sich aus der Abbildungslegende nichts anderes ergibt. Sofern das betreffende Material nicht unter der genannten Creative Commons Lizenz steht und die betreffende Handlung nicht nach gesetzlichen Vorschriften erlaubt ist, ist für die oben aufgeführten Weiterverwendungen des Materials die Einwilligung des jeweiligen Rechteinhabers einzuholen.

Weitere Details zur Lizenz entnehmen Sie bitte der Lizenzinformation auf http://creativecommons.org/ licenses/by/4.0/deed.de.

\section{Literatur}

Assmann A (2017) Formen des Vergessens, 3. Aufl. Wallstein, Göttingen

Bekanntmachung der Neufassung der Richtlinien für die Gewährung von Leistungen wegen Contergan-Schadensfällen; vergl. §8 (2). http:// verwaltungsvorschriften-im-internet.de. Zugegriffen: 3. Sept. 2021

Friedrich C (2005) Contergan - zur Geschichte einer Arzneimittelkatastrophe. In: Zichner L, Rauschmannn MA, Thomann KD (Hrsg) Die Contergankatastrophe - Eine Bilanz nach 40 Jahren. Steinkopff, Heidelberg, S3-12

GemballaG (1993)DerdreifacheSkandal.30 Jahrenach Contergan - eine Dokumentation. Luchterhand, Zürich

Hinoshita F, Beppu H, Shioji Set al (2019) A nationwide survey regarding the life situations of patients with thalidomide embryopathy in Japan, 2018: first report. Birth Defects Res 111(20):1633-1164

Imai K, lida T, Yamamoto M et al (2014) Psychological and mental health problems in patients with thalidomide embryopathy in Japan. Psychiatry Clin Neurosci 68(6):479-486

Kirk B (1999) Der Contergan-Fall: eine unvermeidliche Katastrophe? Zur Geschichte des Arzneistoffs Thalidomid. Greifswalder Schriften zur Geschichte der Pharmazie und Sozialpharmazie, Stuttgart

Knightley P, Evans H, Potter E et al (1979) Suffer the children: the story of thalidomide. André Deutsch Limited, London

Kowalski TW, Sanseverino MT, Schuler-Faccini L et al (2015) Thalidomide embryopathy: follow-up of cases born between 1959 and 2010. Birth Defects Res AClin Mol Teratol 103(9):794-803

\section{Mental health of people with thalidomide embryopathy. Traumatic shadow of prenatal thalidomide exposure}

Background: Sixty years after prenatal thalidomide-induced damage, the clinical picture of people with disabilities caused by thalidomide is characterized by diverse biopsychosocial sequelae.

Objective: How can the high degree of mental health disorders that only become manifest in the second half of life be better understood?

Material and method: Literature review on the current status of empirical research with a discussion of further questions.

Results: People with the most severe thalidomide-induced disabilities showed a remarkable ability to compensate for their disabilities for decades. A very high level of education and good occupational and social integration were achieved over many years. In the more recent past, however, an increasing level of social disintegration has been observed. For the first time, a high rate of mental health disorders has been reported, accompanied by barriers in access to psychotherapeutic care.

Conclusion: The high incidence of mental health disorders in old age can be seen on the one hand as cases of multidimensional traumatization partly already acquired prenatally and on the other hand as a chronic selective disregard of the mental health dimension in favor of a single stigmatization. It is important not only for those directly affected that the case of thalidomide be retained in the collective memory.

\section{Keywords}

Thalidomide - Embryopathy · Physically disabled · Traumatization - Therapeutic remembering

Kruse A, Ding-GreinerC, BeckerGetal (2012)Contergan - Wiederholt durchzuführende Befragungen zu Problemen, speziellen Bedarfen und Versorgungsdefiziten von contergangeschädigten Menschen. Endbericht an die Conterganstiftung für behinderte Menschen. Institut für Gerontologie, Heidelberg

Lehnhard-Schramm N (2016) Das Land NordrheinWestfalen und der Contergan-Skandal. Gesundheitsaufsicht und Strafjustiz in den „langen sechziger Jahren". Vandenhoeck \& Rupprecht, Göttingen

Lenz W (1961) Kindliche Missbildungen nach Medikamenteneinnahme während der Gravidität? Dtsch Med Wochenschr 52:2555-2556

Lenz W (1988) A short history of thalidomide embryopathy. Teratology 38:203-215

Maio G (2017) Mittelpunkt Mensch. Lehrbuch der Ethik in der Medizin, 2. Aufl. Schattauer, Stuttgart, S301

Matthews SJ, McCoy C (2003) Thalidomide: a review of approved and investigational uses. Clin Ther 25(2):342-395

McBride WG (1961) Thalidomide and congenital abnormalities. Letter to the Editor. Lancet 44:1358

Müller-Oerlinghausen B (2005) Die Rolle der Ärzteschaft bei der Aufklärung der Contergannebenwirkungen und die Asuwirkungen auf die deutsche Arzneimittelgesetzgebung. In: Zichner L, Rauschmannn MA, Thomann KD (Hrsg) Die Contergankatastrophe - Eine Bilanz nach 40 Jahren. Steinkopff, Darmstadt, S33-37

Newbronner E, Atkin K (2018) The changing health of thalidomide survivors as they age: a scoping review. Disabil Health J11(2):184-191

Niecke A, Peters K, Samel Cetal (2017) Mental disorders in individuals with thalidomide embryopathy. A cross-sectional study on prevalence and psychosocial healthcare needs. Dtsch Arztebl Int 114(10):168-174
Niecke A, Ramesh I, Albus C et al (2021) Chronische Schmerzen bei Menschen mit ThalidomidEmbryopathie. Eine explorative Analyse zu Prävalenz, schmerzbezogenen Merkmalen und biopsychosozialer Faktoren. Psychother Psychosom Med Psychol 71:e1-e11. https://doi. org/10.1055/a-1457-2846

Oberlander TF, Weinberg J, Papsdorf M et al (2008) Prenatal exposure to maternat depression, neonatal methylation of human glucocortiod receptor gene $\left(\mathrm{NR}_{3} \mathrm{C}_{1}\right)$ and infant cortisol response. Epigenetics 3:97-106

Osten P (2019). Medizinhistorischer Vortrag zum 5jährigen Jubiläum der Contergansprechstunde Schön-KlinikHamburg; 12.12 .2019

Peters KM (2012) Thalidomid-Embryopathie: Eine vielfältige Katastrophe. Orthop Rheuma 15(2):23-28

Peters KM, Albus C, Lüngen Metal (2015) Gesundheitsschäden, psychosoziale Beeinträchtigungen und Versorgungsbedarf von contergangeschädigten Menschen in Nordrhein-Westfalen in der Langzeitperspektive. Forschungsbericht

Schmitz H (2011) Der Leib. De Gruyter, Berlin, Boston

Smithells RW, Newman CGH (1992) Recognition of thalidomide defects. JMed Genet 29:716-723

Stromland K, Nordin V, Miller M et al (1994) Autism in thalidomide embryopathy-a population study. Dev Med Child Neurol 36(4):351-356

Thomann KD (2005) Die Contergan-Epidemie. Ein Beispiel für das Versagen von Staat, Ärzteschaft und Wissenschaft? In: Zichner L, Rauschmannn MA, Thomann KD (Hrsg) Die Contergankatastrophe - Eine Bilanz nach 40 Jahren. Steinkopff, Heidelberg, S13-32

Vargesson N (2009) Thalidomide-induced limb defects: resolving a 50 -year-old puzzle. Bioessays 31(12):1327-1336

Weinrich JM, Beyer R, Well L et al (2018) Assessment of congenital vascular and organ anomalies in subjects with thalidomide embryopathy using 
non-contrast magnetic resonance angiography. Circ J 82(9):2364-2323

Zubin J, Spring B (1977) Vulnerability: a new view of schizophrenia.J Abnorm Psychol 86(2):103-126

Zuckerplätzchen forte (1961) DER SPIEGEL 34. https:// www.spiegel.de.Zugegriffen:3. Sept. 2021

\section{Hier steht eine Anzeige.}

\section{Springer}

